\title{
Epidemiological aspects of the outcomes from the treatment of endometriosis: Experience from two different geographical areas
}

\author{
CHAROULA MATALLIOTAKI ${ }^{1}$, MICHAIL MATALLIOTAKIS ${ }^{1}$, MARIA I. ZERVOU ${ }^{2}$, \\ ATHINA PATELAROU ${ }^{3}$, IOANNIS KOLIARAKIS ${ }^{4}$, DEMETRIOS A. SPANDIDOS ${ }^{5}$, \\ AYDIN ARICI $^{6}$, IOANNIS MATALLIOTAKIS ${ }^{1}$ and GEORGE N. GOULIELMOS ${ }^{2}$
}

\author{
${ }^{1}$ Department of Obstetrics and Gynecology, Venizeleio General Hospital, 71409 Heraklion; ${ }^{2}$ Section of Molecular \\ Pathology and Human Genetics, Department of Internal Medicine, School of Medicine, University of Crete, \\ 71003 Heraklion; ${ }^{3}$ Department of Nursing, School of Health Sciences, Hellenic Mediterranean University, \\ 71410 Heraklion; ${ }^{4}$ Laboratory of Anatomy Histology Embryology, and ${ }^{5}$ Laboratory of Clinical Virology, \\ Medical School, University of Crete, 71003 Heraklion, Greece; ${ }^{6}$ Department of Obstetrics, Gynecology \\ and Reproductive Sciences, Yale University School of Medicine, New Haven, CT 06520, USA
}

Received October 22, 2019; Accepted December 4, 2019

DOI: $10.3892 / \mathrm{etm} .2019 .8296$

\begin{abstract}
The purpose of the present study was two-fold: First to review the epidemiological aspects of the experience on the surgical outcomes via laparotomy or laparoscopy, as regards endometriosis from two different academic institutions and, second, to illustrate potential differences in two different geographical areas, New Haven (US) and Greece. This retrospective study included 1,200 patients (15-80 years of age) treated via laparotomy or laparoscopy, at two different institutions, for endometriosis, between 1990 and 2017. Data were collected and analyzed from medical and pathological reports. The statistical methods used included the Student's t-test and $\chi^{2}$ test, as well as the Mann-Whitney U test. A total of 600 women from Yale University and 600 women from Greece participated in this study. Endometrioma was confirmed in $359(29.9 \%)$ cases. Women were compatible in terms of the site of endometriomas. Left-sided cysts were observed $(\mathrm{P}<0.001)$ significantly more often compared with right-sided cysts in both groups. The two groups of patients had similar rates of endometriosis stages. A statistically significant positive association $(\mathrm{P}<0.001)$ was found for the co-existence of benign gynecological tumors (apart from endometrioma), endometriosis-associated ovarian cancer and for post-menopausal endometriosis in women with endometriosis from Greece. Moreover, similar results were observed as regards endometriosis following in utero exposure to
\end{abstract}

Correspondence to: Dr Michail Matalliotakis, Department of Obstetrics and Gynecology, Venizeleio and Pananio General Hospital of Heraklion, Knossos Avenue, 71409 Heraklion, Greece E-mail: mihalismat@hotmail.com

Key words: endometriosis-associated ovarian cancer, endometrioma, postmenopausal endometriosis diethylstilbestrol (DES), non-Hodgkin's lymphoma, endometriosis-associated Lyme disease, human immuno-deficiency virus (HIV), melanoma and endometriosis in adolescents, between the two groups. To conclude, the two populations exhibited similar results as regards the surgical outcomes of endometriosis laparoscopic or open surgery. Endometriosis represents a multifactorial entity that depends on complex interactions of hormonal, genetic, immunological and environmental factors. Gynecologists should be aware that there is an association between endometriosis and cancerous diseases. It is thus suggested that the presence of comorbidities in women with endometriosis.

\section{Introduction}

Endometriosis is an enigmatic benign common gynecological condition defined as the presence of functional endometrium-like tissue (endometrial glands and stroma) in places outside the uterine cavity, primarily detected in the pelvic peritoneal cavity and ovaries. Although the exact incidence of endometriosis remains unknown, approximately $3-10 \%$ of women of reproductive age suffer from this chronic disease. More than $20 \%$ of women can be asymptomatic and may not undergo abdominal exploration or biopsy procedures. An accurate diagnosis requires the direct visualization of the pelvis during a thorough laparoscopic surgical observation. Based on current knowledge, up to $50 \%$ of women referred for fertility problems, $2-5 \%$ of postmenopausal cases and $10-70 \%$ of patients with pelvic pain are linked to endometriosis. Endometriosis is usually clinically manifested with chronic pelvic pain, dysmenorrhea, dyspareunia and subfertility (1-3).

The complexity of the pathogenesis and the pathophysiology of this disease are the reason for the existence of various controversies in the literature as regards the optimal effective treatment modality for endometriomas. The purpose of surgical management should focus on the confirmation of pre-operative diagnosis, the exclusion of malignant transformation, the 
relief of chronic pelvic pain, dysmenorrhea, dyspareunia or dyschezia and the preservation of future fertility. Surgery for endometriosis and endometrioma can be performed by laparotomy or laparoscopy, as both techniques result in similar symptom regression, fertility treatment, cyst recurrence and major complication rates. However, operative laparoscopy compared with laparotomy represents the gold standard method for the detection of endometriotic implants under magnification in the pelvis. Additionally, the laparoscopic approach offers a lower morbidity, minor post-operative adhesions and a lower duration of hospitalization with a significant better aesthetic result. However, in severe conditions when extensive adhesions or various organs are involved, laparotomy is preferred (4-6). Notably, an association between malignancy and endometriosis has been documented in the literature for a number of years (7). Recently, Kvaskoff et al observed an association of endometriosis, immune dysfunction, and common obstetric and gynecologic diseases (8). Furthermore, Teng et al observed that endometriosis was associated with higher comorbidities (9).

In this study, the authors aimed to investigate the epidemiological aspects of the surgical outcomes of endometriosis surgery from two different academic institutions and to further demonstrate the potential distribution of the diagnosis and important characteristics of endometriosis by institution.

\section{Materials and methods}

The present retrospective study involved 1,200 women (15-80 years of age) treated via laparotomy or laparoscopy, at two different institutions, for endometriosis, between 1990 and 2017. A total of 600 medical records of women with endometriosis were reviewed between 1996-2005, from the Division of Reproductive Endocrinology at Yale University School of Medicine and 600 cases, between 1990 and 2017, from the Department of Obstetrics and Gynecology of the University of Crete and the Department of Venizeleio General Hospital of Heraklion.

The Ethics Committee of Venizeleio Hospital(ECH\#46/6680; ECHR no. 47/773) and the Human Subjects Committee of Yale (HIC\#12590) approved the protocol. The stage of endometriosis was scored according to the revised classification of the American Fertility Society (10) and biopsy specimens were obtained to confirm the histological diagnosis. The following patient characteristics were recorded: Age, age at menarche, main complaints, obstetric outcome, family history, the stage of endometriosis and other important characteristics of endometriosis by institution.

The Student' t-test and $\chi^{2}$ test were used for the comparisons of the mean of the various characteristics. The Mann-Whitney $\mathrm{U}$ test was performed if data were not distributed normally. The results are presented as the means \pm SD or as percentages where appropriate. Differences were considered statistically significant at $\mathrm{P}<0.05$.

\section{Results}

The patients with endometriosis from Greece were older $(\mathrm{P}<0.02)$ in comparison with the women from Yale (Table I). Moreover, infertility $(\mathrm{P}<0.01)$ was mostly observed in patients from Yale and gravida were less frequent among the
Yale population $(\mathrm{P}<0.01)$. All the other characteristics of all the women are presented in Table I. In the Yale and Greek populations, similar results were obtained as regards the site of endometrioma. The occurrence of left-sided endometrioma was found to be significantly higher in both groups.

The distribution of the diagnosis and important characteristics of endometriosis by institution are presented in Table II. No significant differences in endometriosis were observed following in utero exposure to diethylstilbestrol (DES), non-Hodgkin's lymphoma, endometriosis-associated Lyme disease, human immuno-deficiency virus (HIV) and melanoma and endometriosis in adolescents, between the two groups.

A statistically significant positive association $(\mathrm{P}<0.001)$ was noted for the coexistence of benign gynecological tumors (apart from endometrioma), endometriosis-associated ovarian cancer and for postmenopausal endometriosis in women with endometriosis from Greece. However, when patients were analyzed according to the uterine anomalies in association with endometriosis, of note, a higher $(\mathrm{P}<0.05)$ proportion was observed in the Yale population.

\section{Discussion}

Although endometriosis is derived from the ancient Greek words endo (meaning inside), metra (womb) and iosis (meaning infection or viral disease), it was not described as several other gynecological conditions in the writings of Hippocrates, Soranus of Ephesus, or Aesculapius. Daniel Shroen was the first who described endometriosis, 320 years ago in his book entitled 'Disputatio Inauguralis Medica de Ulceritus Ulceri' (11), and has since been accepted as a chronic, painful and often progressive disease affecting women of reproductive age. Endometriosis is an invasive chronic non-cancerous condition and thus, the establishment of extrauterine sites of endometrial implantation requires that endometrial cells are capable of penetrating the mesothelium, or organs within, the peritoneal cavity.

The actual history of endometriosis began after the introduction of endoscopy in the 1970s, where diagnostic laparoscopy was performed for pain and infertility. A PubMed search with the key word, endometriosis, yielded a total of 24,855 articles. Using the combination of surgical endometriosis (laparoscopy and laparotomy) the search yielded 8,410 or $33.8 \%$ of the total literature on endometriosis (accessed January, 2018). It has been well established that endometriosis is still considered a primary surgical disease, despite the relevant accomplishment obtaining using hormone treatment (12). According to current knowledge, surgery represents the only treatment for cystic ovarian endometriosis or deep endometriosis, conditions which are associated with severe pain and infertility. Medical treatment can only inactivate endometriosis without reducing the size of the cyst $(4,6,13)$.

Several retrospective cohort studies have focused on the surgical outcomes of women with endometriosis operated by laparoscopy or laparotomy. These studies have demonstrated that the ideal and most effective conservative laparoscopy technique for the management of endometriosis remains a controversial issue in the literature (14-16). As reported by the literature, cystectomy should be the choice of surgical 
Table I. Distribution of menstrual, clinical and reproductive characteristics of 600 women with endometriosis from Yale center and 600 cases with endometriosis from the Greek population.

\begin{tabular}{|c|c|c|c|}
\hline Characteristic & $\begin{array}{l}\text { Women with endometriosis } \\
\text { from Yale }(\mathrm{n}=600)\end{array}$ & $\begin{array}{l}\text { Women with endometriosis } \\
\text { from Crete }(\mathrm{n}=600)\end{array}$ & P-value ${ }^{a}$ \\
\hline Age in years, median (range) & $32.5 \pm 6.6(15-56)$ & $36.3 \pm 8.2(19-80)$ & $<0.02$ \\
\hline Age at menarche in years, median (range) & $12.2 \pm 1.4(9-18)$ & $12.1 \pm 1.3(10-17)$ & N.S \\
\hline \multicolumn{4}{|l|}{ Main complaints (\%) } \\
\hline Infertility & $325 / 600(54.2 \%)$ & $150 / 600(25 \%)$ & $<0.01$ \\
\hline Pelvic pain & $320 / 600(53.4 \%)$ & $308 / 600(51.3 \%)$ & N.S \\
\hline \multicolumn{4}{|l|}{ Stage of endometriosis } \\
\hline I & $101 / 600(16.8 \%)$ & $95 / 600(15.8 \%)$ & N.S \\
\hline II & $179 / 600(29.8 \%)$ & $169 / 600(28.2 \%)$ & N.S \\
\hline III & $147 / 600(24.5 \%)$ & $161 / 600(26.8 \%)$ & N.S \\
\hline IV & $173 / 600(28.9 \%)$ & $175 / 600(29.2 \%)$ & N.S \\
\hline Endometrioma (\%) & $185 / 600(30.8 \%)$ & $174 / 600(29 \%)$ & N.S \\
\hline Left-sided & $99 / 151(65.5 \%)$ & $94 / 143(65.7 \%)$ & N.S \\
\hline Right-sided & $52 / 151(34.5 \%)^{\mathrm{b}}$ & $49 / 143(34.3 \%)^{\mathrm{b}}$ & N.S \\
\hline Bilateral & 34 & 31 & N.S \\
\hline \multicolumn{4}{|l|}{ Gravida } \\
\hline 0 & $255 / 600(42.5 \%)$ & $91 / 600(15.2 \%)$ & $<0.01$ \\
\hline$\geq 1$ & $345 / 600(57.5 \%)$ & $509(84.8 \%)$ & $<0.01$ \\
\hline Family history of endometriosis & $54 / 600(9 \%)$ & $59 / 600(9.8 \%)$ & N.S \\
\hline
\end{tabular}

Table II. Distribution of the diagnosis and important characteristics of endometriosis by institution.

\begin{tabular}{lccc}
\hline Characteristic & Yale (n=600) & Crete (n=600) & P-value \\
\hline Endometriosis following in utero exposure to DES (\%) & $7 / 600(1.2 \%)$ & $0 / 600(0 \%)$ & N.S \\
Uterine anomalies in women with endometriosis & $19 / 600(3.2 \%)$ & $7 / 600(1.2 \%)$ & $<0.05$ \\
Non-Hodgkin's lymphoma (\%) & $12 / 600(2 \%)$ & $3 / 600(0.5 \%)$ & N.S \\
Endometriosis-associated Lyme disease & $5 / 600(0.9 \%)$ & $1 / 600(0.2 \%)$ & N.S \\
Human immunodeficiency virus (HIV) & $1 / 600(0.2 \%)$ & $2 / 600(0.3 \%)$ & N.S \\
Coexistence of benign gynecological tumors except endometrioma (\%) & $133 / 600(22.1 \%)$ & $265 / 600(44.1 \%)$ & $<0.001$ \\
Endometriosis-associated ovarian cancer & $3 / 600(0.5 \%)$ & $23 / 600(3.9 \%)$ & $<0.001$ \\
Endometriosis and melanoma & $3 / 600(0.5 \%)$ & $2 / 600(0.3 \%)$ & N.S \\
Endometriosis in adolescents (13-20 years old) & $21 / 600(3.5 \%)$ & $29 / 600(4.8 \%)$ & N.S \\
Postmenoposal endometriosis (52-80 years old) & $14 / 600(2.3 \%)$ & $31 / 600(5.2 \%)$ & $<0.001$ \\
\hline
\end{tabular}

Data are presented as n (\%). N.S, not significant.

treatment at the end of the reproductive age or for those with repeated previous operations for endometriosis. Conservative laparoscopic cystectomy seems to be superior to drainage or coagulation in reducing symptoms and recurrence. Although it may damage ovarian follicle reserve, it improves subsequent spontaneous pregnancy rates. Women who experience severe and deep endometriosis and do not have a desire for future pregnancy, are candidates for bilateral salpingo-oophorectomy and hysterectomy.
In the current study, endometrioma was found in 359 (29.9\%) of cases. The two groups of patients had similar rates of the stage of endometriosis and the site of endometrioma. Furthermore, in the Greek population, a coexistence of benign gynecological tumors, endometriosis-associated ovarian cancer and post-menopausal women with endometriosis was observed, which was significant in comparison with the Yale population.

Mutations of the mammalian target of rapamycin (mTOR) signaling pathway have been found to be involved in the 
development of endometrial cancers (17). Contrarily, data have indicated no association of checkpoint kinase 2 (CHEK2), Erb-B2 receptor tyrosine kinase 2 [commonly referred to as HER2; (RBB2)] or ATM with an increased risk of developing endometrial cancer $(18,19)$. In endometrial cancers, somatic mutations are frequent in the MutL homolog 1 (MLH1), MSH2, MutS homolog 6 (MSH6), phosphatase and tensin homolog (PTEN), KRAS and $\beta$-catenin genes in type I carcinomas (20). Although the genetics of benign gynecological tumors (21) and endometriosis-associated ovarian cancer have been extensively studied, genetic analyses focusing on the predisposition for post-menopausal endometriosis are limited. In fact, it is important to be aware of a putative genetic background that may contribute to the development of postmenopausal endometriosis, given that it infers a risk of malignant transformation (22). Postmenopausal women produce estrogen by the steroidogenic gene aromatase that is expressed in stromal cells in endometriosis, and aromatase inhibitors are used to treat postmenopausal endometriosis by suppressing local estrogen production (23). Furthermore, it has been reported that single nucleotide polymorphisms (SNPs) in the DNA base excision repair (BER) system, such as the X-ray repair cross complementing 1 (XRCC1) gene, which has been extensively studied in the association with various types of cancer, harbors an Arg399Gln polymorphism playing an important role in endometrial cancer development in postmenopausal women in a Polish, as well as in a Japanese population $(24,25)$. TP53 Arg72Pro polymorphism has also been reported to be a prognostic factor for endometrial cancer in postmenopausal women (26).

In a previous study, it was observed that endometriosis may be associated with an increased risk of benign gynecological tumors, such as ovarian cysts, adenomyosis and leiomyomas (21). These findings are not surprising, considering that various shared genetic factors have been detected for endometriosis and leiomyoma, i.e., the +2073 A/T polymorphism of the epidermal growth factor receptor $(E G F R)$ gene and the angiotensin I converting enzyme (ACE) I/D polymorphism $(27,28)$ or endometriosis and adenomyosis, i.e., polymorphisms of the catechol- $O$-methyltransferase $(C O M T)$, cytochrome $\mathrm{P} 450$ family 1 subfamily B member 1 (CYP1B1), cyclooxygenase-2 (COX-2), vascular endothelial growth factor $(V E G F)$, matrix metalloproteinase-1 (MMP-1) and MMP-7 genes (29-33). Additionally, the results of the aforementioned study confirmed a left lateral predisposition of endometriomas and ovarian cysts (21). Moreover, apart from the similar co-morbidities described for adenomyosis and endometriosis (34), it has been suggested that the two conditions may have a similar pathogenesis due to a combination of genetic and epigenetic alterations (35), as shown also by data collected upon differential proteomic analysis by iTRAQ technique (36). Taken together, the results suggest that patients suffering from endometriosis should be counseled about the future risks of developing several gynecological diseases.

Furthermore, the present study detected similar results on non-Hodgkin's lymphoma, endometriosis-associated Lyme disease, HIV, melanoma and endometriosis in adolescents between the two groups. Of note, the authors have recently reported an association between endometriosis in adolescents and young women, and risk factors including early menarche, early onset of dysmenorrhea, history of asthma, previous surgical procedures, obstructive genital anomalies, and family history of endometriosis. The demographic observations in this series may reflect a diagnostic bias for endometriosis in the young population who suffers from dysmenorrhea and chronic pelvic pain (37). Notably, several academic studies have noted an association between endometriosis and other severe chronic diseases $(8,9,38,39)$.

In conclusion, the current study reported similar characteristics in both institutions as regards the surgical outcomes on endometriosis surgery. Of note, women with endometriosis have higher co morbidities, including benign and malignant disease. Based on these outcomes, it is thus recommended that clinicians should pay more attention to the combined management of such cases and to further to follow-up all cases operated for endometriosis on a long-term basis, since endometriosis can coexist with other medical diseases.

\section{Acknowledgements}

The authors would like to thank all the clinicians and the pathologists for providing the data and pathology reports used in this study.

\section{Funding}

No funding was received.

\section{Availability of data and materials}

All data generated or analyzed during this study are included in this published article or are available from the corresponding author on reasonable request.

\section{Authors' contributions}

$\mathrm{CM}$ and $\mathrm{MM}$ conceived and designed the study and drafted the manuscript. AA, MIZ and IM obtained the data. GNG, MIZ, CM, AP, DAS and IK analyzed and interpreted the data. GNG, MM, IM and AA revised the manuscript. All authors have read and approved the final manuscript.

\section{Ethics approval and consent to participate}

This is a retrospective study. The Ethics Committee of Venizeleio Hospital (ECH\#46/6680; ECHR no. 47/773) and the Human Subjects Committee of Yale (HIC\#12590) approved the protocol.

\section{Patient consent for publication}

Not applicable.

\section{Competing interests}

DAS is the Editor-in-Chief for the journal, but had no personal involvement in the reviewing process, or any influence in terms of adjudicating on the final decision, for this article. The authors declare no conflict of interest. 


\section{References}

1. Evans MB and Decherney AH: Fertility and Endometriosis. Clin Obstet Gynecol 60: 497-502, 2017.

2. Grimstad FW and Decherney A: A Review of the Epigenetic Contributions to Endometriosis. Clin Obstet Gynecol 60: 467-476, 2017.

3. Somigliana E, Vigano P, Benaglia L, Busnelli A, Berlanda N and Vercellini P: Management of Endometriosis in the Infertile Patient. Semin Reprod Med 35: 31-37, 2017.

4. Zanelotti A and Decherney AH: Surgery and Endometriosis. Clin Obstet Gynecol 60: 477-484, 2017.

5. Tsolakidis D, Pados G, Vavilis D, Athanatos D, Tsalikis T, Giannakou A and Tarlatzis BC: The impact on ovarian reserve after laparoscopic ovarian cystectomy versus three-stage management in patients with endometriomas: A prospective randomized study. Fertil Steril 94: 71-77, 2010.

6. Alborzi S, Hosseini-Nohadani A, Poordast T and Shomali Z: Surgical outcomes of laparoscopic endometriosis surgery: A 6 year experience. Curr Med Res Opin 33: 2229-2234, 2017.

7. Brinton LA, Gridley G, Persson I, Baron J and Bergqvist A: Cancer risk after a hospital discharge diagnosis of endometriosis. Am J Obstet Gynecol 176: 572-579, 1997.

8. Kvaskoff M, Mu F, Terry KL, Harris HR, Poole EM, Farland L and Missmer SA: Endometriosis: A high-risk population for major chronic diseases? Hum Reprod Update 21: 500-516, 2015.

9. Teng SW, Horng HC, Ho CH, Yen MS, Chao HT, Wang PH, Chang YH, Chang Y, Chao KC, Chen YJ, et al; Taiwan Association of Gynecology Systematic Review Group: Women with endometriosis have higher comorbidities: Analysis of domestic data in Taiwan. J Chin Med Assoc 79: 577-582, 2016.

10. American Society for Reproductive: Revised American Society for Reproductive Medicine classification of endometriosis: 1996 Fertil Steril 67: 817-821, 1997.

11. Shroen D: Disputatio inauguralis medica de ulceritus ulceri. Jena, Krebs, pp6-17, 1690).

12. Somigliana E, Vercellini P, Vigano P, Benaglia L, Busnelli A and Fedele L: Postoperative medical therapy after surgical treatment of endometriosis: From adjuvant therapy to tertiary prevention. J Minim Invasive Gynecol 21: 328-334, 2014.

13. Fawole AO, Bello FA, Ogunbode O, Odukogbe AT, Nkwocha GC, Nnoaham KE, Zondervan KT, Akintan A, Abdus-Salam RA and Okunlola MA: Endometriosis and associated symptoms among Nigerian women. Int J Gynaecol Obstet 130: 190-194, 2015.

14. Vercellini P, Chapron C, De Giorgi O, Consonni D, Frontino G and Crosignani PG: Coagulation or excision of ovarian endometriomas? Am J Obstet Gynecol 188: 606-610, 2003.

15. Alborzi S, Zarei A, Alborzi S and Alborzi M: Management of ovarian endometrioma. Clin Obstet Gynecol 49: 480-491, 2006.

16. Dilek U, Pata O, Tataroglu C, Aban M and Dilek S: Excision of endometriotic cyst wall may cause loss of functional ovarian tissue. Fertil Steril 85: 758-760, 2006.

17. Bajwa P, Nielsen S, Lombard JM, Rassam L, Nahar P, Rueda BR, Wilkinson JE, Miller RA and Tanwar PS: Overactive mTOR signaling leads to endometrial hyperplasia in aged women and mice. Oncotarget 8: 7265-7275, 2017.

18. Einarsdóttir K, Humphreys K, Bonnard C, Li Y, Li Y, Chia KS, Liu ET, Hall P, Liu J and Wedrén S: Effect of ATM, CHEK2 and ERBB2 TAGSNPs and Haplotypes on Endometrial Cancer Risk. Hum Mol Genet 16: 154-164, 2007.

19. Einarsdóttir K. Humphreys K, Bonnard C, Palmgren J, Iles MM, Sjölander A, Li Y, Chia KS, Liu ET, Hall P, et al: Linkage disequilibrium mapping of CHEK2: Common variation and breast cancer risk. PLoS Med 3: e168, 2006.

20. Carter J and Pather S: An overview of uterine cancer and its management. Expert Rev Anticancer Ther 6: 33-42, 2006.

21. Matalliotaki C, Matalliotakis M, Ieromonachou P, Goulielmos GN, Zervou MI, Laliotis A, Spandidos DA, Arici A and Matalliotakis I: Co-existence of benign gynecological tumors with endometriosis in a group of 1,000 women. Oncol Lett 15: 1529-1532, 2018.

22. Oxholm D, Knudsen UB, Kryger-Baggesen N and Ravn P: Postmenopausal endometriosis. Acta Obstet Gynecol Scand 86: 1158-1164, 2007.

23. Attar E and Bulun SE: Aromatase and other steroidogenic genes in endometriosis: Translational aspects. Hum Reprod Update 12: 49-56, 2006.
24. Romanowicz-Makowska H, Smolarz B, Góralczyk B, Mroziewicz K, Połać I, Szyłł K and Kulig A: hOGG1 Ser326Cys and XRCC1 Arg399Gln genetics polymorphism in DNA repair genes by base excision repair pathway (BER) in postmenopausal women with endometrial cancer. Przegl Menopauz 6: 366-370, 2010.

25. Hosono S, Matsuo K, Ito H, Oze I, Hirose K, Watanabe M, Nakanishi T, Tajima K and Tanaka H: Polymorphisms in base excision repair genes are associated with endometrial cancer risk among postmenopausal Japanese women. Int J Gynecol Cancer 23: 1561-1568, 2013.

26. Zając A, Stachowiak G, Smolarz B and Wilczyński JR: Polymorphisms of codon 72 of the TP53 gene in endometrial carcinoma of postmenopausal women. Postepy Hig Med Dosw 67: 1312-1318, 2013.

27. Hsieh YY, Chang CC, Tsai FJ, Lin CC and Tsai CH: T homozygote and allele of epidermal growth factor receptor 2073 gene polymorphism are associated with higher susceptibility to endometriosis and leiomyomas. Fertil Steril 83: 796-799, 2005.

28. Hsieh YY, Lee CC, Chang CC, Wang YK, Yeh LS and Lin CS: Angiotensin I-converting enzyme insertion-related genotypes and allele are associated with higher susceptibility of endometriosis and leiomyoma. Mol Reprod Dev 74: 808-814, 2007.

29. Kang S, Wang Y, Zhang JH, Jin X, Fang SM and Li Y: Single nucleotide polymorphism in the matrix metalloproteinases promoter is associated with susceptibility to endometriosis and adenomyosis. Zhonghua Fu Chan Ke Za Zhi 40: 601-604, 2005 (In Chinese).

30. Shan K, Lian-Fu Z, Hui D, Wei G, Na W, Xia J and Yan L: Polymorphisms in the promoter regions of the matrix metalloproteinases-7, -9 and the risk of endometriosis and adenomyosis in China. Mol Hum Reprod 12: 35-39, 2006.

31. Liu Q, Li Y, Zhao J, Zhou RM, Wang N, Sun DL, Duan YN and Kang S: Association of single nucleotide polymorphisms in VEGF gene with the risk of endometriosis and adenomyosis. Zhonghua Yi Xue Yi Chuan Xue Za Zhi 26: 165-169, 2009 (In Chinese).

32. Tong X, Li Z, Wu Y, Fu X, Zhang Y and Fan H: COMT 158G/A and CYP1B1 $432 \mathrm{C} / \mathrm{G}$ polymorphisms increase the risk of endometriosis and adenomyosis: A meta-analysis. Eur J Obstet Gynecol Reprod Biol 179: 17-21, 2014.

33. Wang Y, Qu Y and Song W: Genetic variation in COX-2 -1195 and the risk of endometriosis and adenomyosis. Clin Exp Obstet Gynecol 42: 168-172, 2015.

34. Choi EJ, Cho SB, Lee SR, Lim YM, Jeong K, Moon HS and Chung H: Comorbidity of gynecological and non-gynecological diseases with adenomyosis and endometriosis. Obstet Gynecol Sci 60: 579-586, 2017.

35. Koninckx PR, Ussia A, Zupi E and Gomel V: Association of Endometriosis and Adenomyosis: Vast Literature but Scant Conclusive Data. J Minim Invasive Gynecol 25: 745-748, 2018.

36. Xiaoyu L, Weiyuan Z, Ping J, Anxia W and Liane Z: Serum differential proteomic analysis of endometriosis and adenomyosis by iTRAQ technique. Eur J Obstet Gynecol Reprod Biol 182: 62-65, 2014.

37. Matalliotakis M, Goulielmos GN, Matalliotaki C, Trivli A, Matalliotakis I and Arici A: Endometriosis in adolescents and young girls: Report a series of 85 cases. J Pediatr Adolesc Gynecol 30: 568-570, 2017.

38. Sinaii N, Cleary SD, Ballweg ML, Nieman LK and Stratton P: High rates of autoimmune and endocrine disorders, fibromyalgia, chronic fatigue syndrome and atopic diseases among women with endometriosis: A survey analysis. Hum Reprod 17: 2715-2724, 2002.

39. Matalliotaki C, Matalliotakis M, Zervou MI, Trivli A, Matalliotakis I, Mavromatidis G, Spandidos DA, Albertsen HM, Chettier R, Ward K, et al: Co-existence of endometriosis with 13 non-gynecological co-morbidities: Mutation analysis by whole exome sequencing. Mol Med Rep 18: 5053-5057, 2018.

This work is licensed under a Creative Commons Attribution-NonCommercial-NoDerivatives 4.0 International (CC BY-NC-ND 4.0) License. 